\title{
REVIEW OF THE N-QUANTUM APPROACH TO BOUND STATES
}

\author{
O.W. Greenberg 1 \\ Center for Fundamental Physics \\ Department of Physics \\ University of Maryland \\ College Park, MD 20742-4111, US \\ and Helsinki Institute of Physics \\ P.O.Box 64 \\ FIN-00014 University of Helsinki \\ Finland \\ University of Maryland Preprint PP-10021
}

\begin{abstract}
We describe a method of solving quantum field theories using operator techniques based on the expansion of interacting fields in terms of asymptotic fields. For bound states, we introduce an asymptotic field for each (stable) bound state. We choose the nonrelativistic hydrogen atom as an example to illustrate the method. Future work will apply this N-quantum approach to relativistic theories that include bound states in motion.
\end{abstract}

\section{Introduction}

We describe a method for solving of quantum field theories (QFT) that has wide applicability, and is analogous to methods developed to solve problems in classical theories. To find approximate solutions of a theories with a given Lagrangian, the method approximates the Lagrangian fields by a series of normal-ordered asymptotic fields that terminate with a product of up to $N$ asymptotic fields. This $N$ is the "N" of the N-quantum approximation. R. Haag introduced this expansion [1. We modified his work by introducing asymptotic fields for the stable bound states, if

\footnotetext{
${ }^{1}$ email address, owgreen@umd.edu
} 
any, in the theory. We derive analogs of the Schrödinger equation that have solutions when the theory has bound states.

We apply this method to the hydrogen atom to illustrate how this works for bound states. Following C.N. Yang and D. Feldman [2], we use the QFT analog of the solution of classical equations of motion in which the the classical equations govern a set of functions while the QFT equations of motion govern a set of operators. As stated above we take account of the operator aspect of quantum field theory by using a complete and irreducible set of asymptotic fields, either the in or the out fields [1]. A complete set of fields is one that creates all the states of the Hilbert space by acting repeatedly on a given vector, often the vacuum, called the cyclic vector. (More accurately, the complete set needs only to approximate any vector when polynomials in the operators, integrated ("smeared") using smooth functions, act on the cyclic vector.) An irreducible set of fields is a set that has no proper invariant subspace. By Schur's lemma, any operator that commutes with all operators in an irreducible set must be a multiple of the identity operator. Saying that the in (or out) fields comprise a complete (and irreducible) set, means that any operator in the theory can be expressed in terms of them.

The in (or out) fields have free-field commutators, obey free equations of motion, and the different in (or out) fields commute or anticommute with each other everywhere in spacetime. Each of these sets of asymptotic fields is completely known once the masses, spins and quantum numbers of the fields in a given set are defined. Thus, either set serves as a collection of standard building blocks from which we can construct solutions of the operator equations of motion.

To solve the equations of motion using the Yang-Feldman approach, we expand the fields that appear in the Hamiltonian or Lagrangian in normal-ordered series of in (or out) fields. (This is the Haag expansion.) To determine the c-number amplitudes (the Haag amplitudes) that are the coefficients of the normal-ordered terms, we insert the expansion in the operator equations of motion, again normalorder the asymptotic fields, and equate the coefficients of corresponding (linearly independent) normal-ordered terms.

In this paper, we illustrate this technique for the case of the nonrelativistic hydrogen atom [3]. The amplitudes for the bound states satisfy the Schrödinger 
equation for the hydrogen atom and, of course, we find the usual spectrum of bound states. This shows that the relevant Haag amplitudes are the wave functions of the bound states.

Our method, which is based on the Haag expansion, is entirely independent of the Bethe-Salpeter equation. In contrast to the Bethe-Salpeter approach, our method contains no spurious solutions and no negative norm amplitudes. The method can be used for bound states in relativistic theories, despite that the amplitudes depend on the same number of kinematic variables as appear in nonrelativistic wave functions. There are no relative time intervals in the relativistic version of this method.

We applied this formulation previously to a model of the deuteron [4] in an approximation where this method is similar to that of the Gross equation [5], but differs from it in higher-order approximations.

In this paper, we neglect two related issues that are relevant for the Coulomb interaction. One is that, because the Coulomb interaction is long ranged, spatially separated particles never move independently [6]. This does affect any questions addressed in our study. The other point is that the Coulomb field of charged particles is not attached to the particles, as suggested by P.A.M. Dirac [7]. Nevertheless, this too does not appear to cause any difficulty for the conclusions drawn from this work.

Although our method does not require expressions for interpolating (Heisenberg) in and out asymptotic fields for bound states, we show how to construct such interpolating fields for the bound states of the theory (in our case, the states of the hydrogen atom) in terms of the fundamental fields that enter the Hamiltonian of the theory. This construction, which uses a convolution of the bound-state wave functions with the constituent fields, contrasts with the constructions of K. Nishijima [8] and of W. Zimmermann [9], which use products of the fundamental fields at the same point.

We plan to apply the construction of interpolating fields for bound states of baryons and mesons to calculations based on quark models within quantum chromodynamics.

We note that when we use in (out) fields as the asymptotic fields, we have retarded (advanced) boundary conditions on the amplitudes (Green's functions) that 
provide dispersion relations for these amplitudes, which G. Källén [10] discussed in the context of perturbation theory for Yang-Feldman equations with retarded boundary conditions.

\section{Asymptotic fields and the Haag expansion}

Asymptotic fields have been part of quantum field theory at least since the work of H. Lehmann, K. Symanzik and W. Zimmermann [11]; nevertheless, there are still misconceptions that should be cleared up. Asymptotic fields are free fields in theories that have no long-range potentials, massless particles or confinement, because, in

such theories, we describe particles either by: (i) separated asymptotic fields (at large time scales) or (ii) as a bound state, also represented by an independent asymptotic field. In either case, the exact eigenstates can be labeled by the quantum numbers of the free particles. The (weak) limits for $t \rightarrow \pm \infty$ are the out or in fields that construct the eigenstates at the corresponding limiting times [12]. The nontrivial unitary relation between these fields is given by the $S$-operator,

$$
S \phi^{\text {out }}(x) S^{\dagger}=\phi^{\text {in }}(x)
$$

The asymptotic fields at finite times are the limiting fields propagated to finite times according to the free equations of motion. The free-field property of the asymptotic fields does not depend on any unphysical "adiabatic switching off" of interactions. Even when the potentials are long-range (and there are massless particles in the context of the field theory), we can use often use the usual asymptotic fields. as is the case for the example considered in this paper.

The limits that define the asymptotic fields are subtle. The relations that appear in some books,

$$
A(\mathbf{x}, t) \rightarrow A^{(\text {out }) i n}(\mathbf{x}, t), \quad t \rightarrow \pm \infty
$$

are ill-defined. We discuss the proper (weak-limit) definition of the asymptotic fields in the Appendix. 


\section{Nonrelativistic model of the hydrogen atom}

The fundamental fields are the spin $1 / 2$ electron, $e_{a}(\mathbf{x}, t)$, and the spin $1 / 2$ proton, $p_{a}(\mathbf{x}, t)$, fields. The $a$ indices label the spin components of the fields. We assume that these fields obey the usual equal-time canonical anticommutation relations,

$$
\begin{aligned}
& {\left[e_{a}(\mathbf{x}, t), e_{b}^{\dagger}(\mathbf{y}, t)\right]_{+}=\delta_{a b} \delta(\mathbf{x}-\mathbf{y})} \\
& {\left[p_{a}(\mathbf{x}, t), p_{b}^{\dagger}(\mathbf{y}, t)\right]_{+}=\delta_{a b} \delta(\mathbf{x}-\mathbf{y})}
\end{aligned}
$$

and that the other equal-time anticommutators vanish. The nonrelativistic Hamiltonian is

$$
\begin{aligned}
H= & \frac{1}{2 m} \int d^{3} x \nabla_{\mathbf{x}} e_{a}^{\dagger}(\mathbf{x}, t) \cdot \nabla_{\mathbf{x}} e_{a}(\mathbf{x}, t)+\frac{1}{2 M} \int d^{3} x \nabla_{\mathbf{x}} p_{a}^{\dagger}(\mathbf{x}, t) \cdot \nabla_{\mathbf{x}} p_{a}(\mathbf{x}, t) \\
& -\int d^{3} x d^{3} y p_{b}^{\dagger}(\mathbf{y}, t) e_{a}^{\dagger}(\mathbf{x}, t) \frac{e^{2}}{|\mathbf{x}-\mathbf{y}|} e_{a}(\mathbf{x}, t) p_{b}(\mathbf{y}, t),
\end{aligned}
$$

where $m$ and $M$ are the electron and proton masses. (We have not included spindependent terms in the interaction.) The equation of motion for the electron field is

$$
i \partial_{t} e_{a}(\mathbf{x}, t)=-\frac{1}{2 m} \nabla_{\mathbf{x}}^{2} e_{a}(\mathbf{x}, t)-\int d^{3} y p_{b}^{\dagger}(\mathbf{y}, t) p_{b}(\mathbf{y}, t) \frac{e^{2}}{|\mathbf{x}-\mathbf{y}|} e_{a}(\mathbf{x}, t)
$$

The proton field obeys a similar equation.

\section{Derivation of the Schrödinger equation for the hydrogen atom}

We illustrate the N-quantum approach for the hydrogen atom in the approximation in which the excited states of hydrogen are stable. We introduce the terms for this case into the Haag expansions of the electron and proton fields. The relevant in fields (alternatively, the out fields can be used as well) are those for the electron, $e_{a}^{i n}(\mathbf{x}, t)$, the proton, $p_{a}^{i n}(\mathbf{x}, t)$ and the in fields for each state of the hydrogen atom, $h_{n^{\prime} l^{\prime} m^{\prime}}^{i n}$. For simplicity, we choose the $F=0$ state of the hydrogen atom, so the in field, $h_{n^{\prime} l^{\prime} m^{\prime}}^{i n}$, is a scalar boson. (We use the known spectrum of the hydrogen 
atom. In general, we would use the Schrödinger equation, which we derive below, to find any such bound states.) The in fields obey free equations of motion and free anticommutation (commutation) relations: For the electron, we have,

$$
\begin{gathered}
i \partial_{t} e_{a}^{i n}(\mathbf{x}, t)=-\frac{1}{2 m} \nabla_{\mathbf{x}}^{2} e_{a}^{i n}(\mathbf{x}, t) \\
{\left[e_{a}^{i n}(\mathbf{x}, t), e_{b}^{i n \dagger}\left(\mathbf{y}, t^{\prime}\right)\right]_{+}=\delta_{a b} \mathcal{D}\left(\mathbf{x}-\mathbf{y}, t-t^{\prime} ; 0, m\right),} \\
\mathcal{D}(\mathbf{x}, t ; E, m)=\frac{1}{(2 \pi)^{3}} \int d \omega d^{3} k \delta\left(\omega-E-\frac{\mathbf{k}^{2}}{2 m}\right) e^{-i \omega t+i \mathbf{k} \cdot \mathbf{x}} .
\end{gathered}
$$

The proton in field obeys the analogous equations, with the proton mass replacing the electron mass. The in fields for the states of the hydrogen atom obey,

$$
\begin{gathered}
i \partial_{t} h_{n l m}^{i n}(\mathbf{x}, t)=\left(E_{n l m}-\frac{1}{2 M_{n l m}} \nabla_{\mathbf{x}}^{2}\right) h_{n l m}^{i n}(\mathbf{x}, t), \\
{\left[h_{n l m}^{i n}(\mathbf{x}, t), h_{n^{\prime} l^{\prime} m^{\prime}}^{i n \dagger}\left(\mathbf{y}, t^{\prime}\right)\right]_{-}=\delta_{n l m, n^{\prime} l^{\prime} m^{\prime}} \mathcal{D}\left(\mathbf{x}-\mathbf{y}, t-t^{\prime} ; E_{n l m}, M_{n l m}\right),}
\end{gathered}
$$

where $M_{n l m}=m+M+E_{n l m}$ is the mass of the hydrogen atom in the state $n l m$. The anticommutators or commutators between different in fields vanish.

Because the electron is stable the appropriate terms in the Haag expansion for the electron field are the electron in field, and for the states of the hydrogen atom, the hydrogen in fields. In the annihilation part for the electron, the operators that enter the bound-state term are the hydrogen annihilation operator and the creation operator for the proton. The quantum numbers balance, in that, for example, both $e^{i n}$ and $p^{i n \dagger} h_{n l m}^{i n}$ annihilate charge $-e$ and lepton number +1 . (For simplicity, we choose the $F=0$ state of the hydrogen atom, so that $h_{n l m}^{\text {in }}$ represents a scalar boson.) As will be shown explicitly below, this term in the Haag amplitudes corresponds to the usual bound-state wave functions for the hydrogen atom. We choose a scalar hydrogen atom to simplify our discussion. Using the constraint [13] from the nonrelativistic boost, $\mathbf{k} \rightarrow \mathbf{k}+\mathbf{v}$, we find

$$
\begin{aligned}
e_{a}(\mathbf{x}, t)= & e_{a}^{i n}(\mathbf{x}, t) \\
& +\sum_{n l m} \int \psi_{n l m}^{e}(\mathbf{x}-\mathbf{y}) \epsilon_{a b}: p_{b}^{i n \dagger}(\mathbf{y}, t) h_{n l m}^{i n}\left(\frac{m \mathbf{x}+M \mathbf{y}}{m+M}, t\right): d^{3} y,
\end{aligned}
$$


The analogous expansion holds for the proton field:

$$
\begin{aligned}
p_{a}(\mathbf{y}, t)= & p_{a}^{i n}(\mathbf{y}, t) \\
& +\sum_{n l m} \int \psi_{n l m}^{p}(\mathbf{x}-\mathbf{y}) \epsilon_{a b}: e_{b}^{i n \dagger}(\mathbf{y}, t) h_{n l m}^{i n}\left(\frac{m \mathbf{x}+M \mathbf{y}}{m+M}, t\right): d^{3} y .
\end{aligned}
$$

The wave function $\psi_{n l m}^{e}$ is the matrix element of the Hamiltonian field $e_{a}(\mathbf{x}, t)$ between the proton in state and the in states for the hydrogen atom:

$$
{ }_{i n}\left\langle p_{b}(\mathbf{y}, t)\left|e_{a}(\mathbf{x}, t)\right| h(\mathbf{X}, t)\right\rangle_{i n}=\epsilon_{a b} \psi_{n l m}^{e}(\mathbf{x}-\mathbf{y}) \delta\left(\frac{m \mathbf{x}+M \mathbf{y}}{m+M}-\mathbf{X}\right) .
$$

To find the equation for the wave function $\psi_{n l m}^{e}$, we substitute these expansions into the equation of motion for the electron field, again normal-order the in fields, and examine the terms that contain $p_{b}^{i n \dagger} h_{n l m}^{i n}$ :

$$
\begin{array}{r}
i \partial_{t} \int \sum_{n l m} \psi_{n l m}^{e}(\mathbf{x}-\mathbf{y}, \mathbf{x}-\mathbf{X}) \epsilon_{a b}: p_{b}^{i n \dagger}(\mathbf{y}, t) h_{n l m}^{i n}\left(\frac{m \mathbf{x}+M \mathbf{y}}{m+M}, t\right): d^{3} y= \\
\sum_{n l m} \int\left(-\frac{1}{2 m} \nabla_{\mathbf{x}}^{2}-\frac{e^{2}}{|\mathbf{x}-\mathbf{y}|}\right) \psi_{n l m}^{e}(\mathbf{x}-\mathbf{y}) \epsilon_{a b}: p_{b}^{i n \dagger}(\mathbf{y}, t) h_{n l m}^{i n}\left(\frac{m \mathbf{x}+M \mathbf{y}}{m+M}, t\right): d^{3} y
\end{array}
$$

To find the Schrödinger equation for the hydrogen atom with momentum $\mathbf{P}$, we introduce the Fourier transform:

$$
h_{n l m}^{i n}(\mathbf{x}, t)=\frac{1}{(2 \pi)^{3 / 2}} \int \tilde{h}^{i n}(\mathbf{P}) \exp \left(-i\left(E_{n l m}+\frac{\mathbf{P}^{2}}{2 M_{n l m}}\right) t+i \mathbf{P} \cdot \mathbf{x}\right) d^{3} P .
$$

In terms of the Fourier transform, the equation is

$$
\begin{aligned}
& \sum_{n l m} \int \psi_{n l m}^{e}(\mathbf{x}-\mathbf{y}) \epsilon_{a b}\left(i \partial_{t}+E_{n l m}+\frac{\mathbf{P}^{2}}{2 M_{n l m}}\right): p_{b}^{i n \dagger}(\mathbf{y}, t) \tilde{h}_{n l m}^{i n}(\mathbf{P}): d^{3} y= \\
& \sum_{n l m} \int\left(-\frac{1}{2 m} \nabla_{\mathbf{x}}^{2}-\frac{e^{2}}{|\mathbf{x}-\mathbf{y}|}\right) \psi_{n l m}^{e}(\mathbf{x}-\mathbf{y}) \epsilon_{a b}: p_{b}^{i n \dagger}(\mathbf{y}, t) \tilde{h}_{n l m}^{i n}(\mathbf{P}): d^{3} y .
\end{aligned}
$$

We evaluate the time derivative on the left hand side using the equation for the $p_{b}^{i n \dagger}$, and integrate by parts to get derivatives acting on the wave function. The 
left hand side becomes

$$
\left.\sum_{n l m} \int\left(\frac{1}{2 M} \nabla_{\mathbf{y}}^{2}+E_{n l m}+\frac{\mathbf{P}^{2}}{2 M_{n l m}}\right) \psi_{n l m}^{e}(\mathbf{x}-\mathbf{y}) \epsilon_{a b}: p_{b}^{i n \dagger}(\mathbf{y}, t)\right) \tilde{h}_{n l m}^{i n}(\mathbf{P}): d^{3} y .
$$

We do not assume the hydrogen atom is at rest; the last term above gives its kinetic energy. We are using the kinematics for the nonrelativistic limit of a Lorentz covariant theory. (For a Galilean covariant theory, the mass of the hydrogen atom is $m+M$ for all states of the hydrogen atom because of the Bargmann superselection rule. [14]) The final step is to equate the coefficients of the $p_{b}^{i n \dagger} \tilde{h}_{n l m}^{i n}$ terms. We justify this either by noting that these terms are linearly independent of all other types of in field products or, more formally, by anticommuting with $p_{b}^{i n}$ and commuting with $h_{n l m}^{i n}$. The result is

$$
\begin{gathered}
\left.\left(-\frac{1}{2 m} \nabla_{\mathbf{x}}^{2}-\frac{1}{2 M} \nabla_{\mathbf{y}}^{2}-\frac{e^{2}}{|\mathbf{x}-\mathbf{y}|}\right) \psi_{n l m}^{e}(\mathbf{x}-\mathbf{y})=\left(E_{n l m}+\frac{\mathbf{P}^{2}}{2 M_{n l m}}\right) \psi_{n l m}^{e}(\mathbf{x}-\mathbf{y})\right) \\
E_{n l m}=-\frac{m M e^{4}}{2(m+M) n^{2}}
\end{gathered}
$$

where we used the known energy levels of the hydrogen atom. We recognize this as the Schrödinger equation for the hydrogen atom and identify the $\psi_{n l m}^{e}$ as its wave functions.

In terms of the relative coordinate, $\mathbf{r}=\mathbf{x}-\mathbf{y}$, the Schrödinger equation for the hydrogen atom with momentum $\mathbf{P}$ is

$$
\left(-\frac{1}{\mu_{n l m}} \nabla_{\mathbf{r}}^{2}-\frac{e^{2}}{|\mathbf{r}|}\right) \psi_{n l m}^{e}(\mathbf{r})=\left(E_{n l m}+\frac{1}{\mu_{n l m}} \mathbf{P}^{2}\right) \psi_{n l m}^{e}(\mathbf{r}),
$$

where $1 / \mu=1 / m+1 / M$ and $1 / \mu_{n l m}=1 / M_{n l m}-1 /(m+M)$.

Because $1 / \mu_{n l m}>0$, the relative wave function for the bound state in motion obeys an equation with a (slightly) higher energy than that for the bound state at rest. In a Galilean invariant theory, $M_{n l m}=M+m$ for all states of the hydrogen atom, and the atom in motion obeys the same equation as the atom at rest because of the Bargmann mass superselection rule [14]. 
The bound states of the hydrogen atom, taking into account the spin of the electron and the proton, are labeled by $n L S J J_{z}$, and the $\mathrm{N}$ quantum amplitudes also have these labels, as well as spin indices $a, b$ for the electron and proton spins. For the $S=0$ states, the amplitude is

$$
\psi_{a b J_{z}}^{e J}(n L S=0)(\mathbf{r})=\epsilon_{a b} \psi_{J_{z}}^{e J}(n L S=0)(\mathbf{r}) .
$$

For the $S=1$ states the amplitude is

$$
\psi_{a b J z}^{e J}(n L S=1)(\mathbf{r})=\sigma_{a b}^{S_{z}} \psi_{J_{z}}^{e J}(n L S=1)(\mathbf{r}),
$$

where $J=L-1, L, L+1$. The corresponding annihilation operators for the $S=0$ hydrogen atom are

$$
h_{J_{z}}^{J}(n L S, J=L ; \mathbf{R})=h_{L_{z}, J_{z}=L_{z}}^{L S=0}(\mathbf{R})
$$

and for $S=1$,

$$
\begin{aligned}
& h_{J_{z}}^{J}(n L S, J=L-1, L, L+1 ; \mathbf{R})= \\
& \quad \sum_{L_{z}} \sqrt{2 J+1}(-)^{L-S-J_{z}}\left(\begin{array}{ccc}
L & S & J \\
L_{z} & J_{z}-L_{z} & J_{z}
\end{array}\right) h_{L_{z}, J_{z}-L_{z}=S_{z}}^{L S}(\mathbf{R}) .
\end{aligned}
$$

\section{Normalization of the wave functions}

The asymptotic fields diagonalize conserved observables such as the Hamiltonian, the momentum operators and various charges. Thus, any such conserved quantity has the form of a sum of free operators, $\mathcal{O}_{\text {free }}\left\{\phi_{i n}\right\}$, for the contribution of each in field, including those for bound states, to the observable $\mathcal{O}$. For the $\psi_{n l m}^{e}$, with the electron off-shell, we choose the lepton number $L$, whose term in the in field expansion provides the normalization condition, to be the observable. The lepton number in this model is

$$
L=\int d^{3} x e^{\dagger}(\mathbf{x}, 0) e(\mathbf{x}, 0)=L_{0}\left\{e^{i n}\right\}+\sum_{n l m} L_{0}\left\{h_{n l m}^{i n}\right\}+\cdots .
$$

We substitute the Haag expansion for $e(\mathbf{x}, t)$ given in Eq.(12) into $L$, look for the terms bilinear in $h_{n l m}^{i n}$, and equate these to $L_{0}\left\{h_{n l m}^{i n}\right\}=\int d^{3} R h_{n l m}^{i n \dagger} h_{n l m}^{i n}$. We find

$$
\int d^{3} x \sum_{n l m} \psi_{n l m}^{e *}(\mathbf{x}-\mathbf{y}) h_{n l m}^{i n \dagger}(\mathbf{R}, 0) p^{i n}(\mathbf{y}, 0) d^{3} y
$$




$$
\begin{aligned}
& \times \sum_{n^{\prime} l^{\prime} m^{\prime}} \psi_{n^{\prime} l^{\prime} m^{\prime}}^{e}\left(\mathbf{x}-\mathbf{y}^{\prime}\right) p^{i n \dagger}\left(\mathbf{y}^{\prime}, 0\right) h_{n^{\prime} l^{\prime} m^{\prime}}^{i n}\left(\mathbf{R}^{\prime}, 0\right) d^{3} y^{\prime}= \\
& \sum_{n l m} \int d^{3} R h_{n l m}^{i n \dagger}(\mathbf{R}, 0) h_{n l m}^{i n}(\mathbf{R}, 0)
\end{aligned}
$$

with $R=(m \mathbf{x}+M \mathbf{y}) /(m+M)$ and $R^{\prime}=\left(m \mathbf{x}+M \mathbf{y}^{\prime}\right) /(m+M)$. The $p^{\text {in }}$ operators on the left-hand side contract, $\left\langle p^{i n}(\mathbf{y}, 0) p^{i n \dagger}\left(\mathbf{y}^{\prime}, 0\right)\right\rangle=\delta\left(\mathbf{y}-\mathbf{y}^{\prime}\right)$. When we remove the $h_{n l m}^{\text {in }}$ operators by commuting with $h_{n l m}^{\text {in }}$ operators, the usual orthonormalization condition results:

$$
\int d^{3} r \psi_{n l m}^{e *}(\mathbf{r}) \psi_{n^{\prime} l^{\prime} m^{\prime}}^{e}(\mathbf{r})=\delta_{n l m, n^{\prime} l^{\prime} m^{\prime}}
$$

\section{Equal-time anticommutation relations}

The equal-time anticommutation relations give relations among the Haag amplitudes. These relations follow from the vanishing of the coefficients of each (linearly independent) normal-ordered product of in fields. Most of the relations involve Haag amplitudes for terms with higher-degree normal-ordered products than we have considered, however, for the equal-time anticommutator $[e, p]_{+}=0$, there is a relation that involves the wave functions:

$$
\psi_{n l m}^{e}(\mathbf{r})+\psi_{n l m}^{p}(-\mathbf{r})=0
$$

Thus the Haag amplitude for the off-shell electron is related simply to that for the off-shell proton, and the apparent asymmetry in the treatment of the constituents of the bound state because one particle is on-shell and one particle is off-shell is therefore not a true asymmetry. The two amplitudes determine each other uniquely, and it is convenient to define $\psi_{n l m}(\mathbf{r}) \equiv \psi_{n l m}^{e}(\mathbf{r})$.

\section{Definition of asymptotic limits}

The proper asymptotic limit is a weak operator limit that constructs an asymptotic field of a given mass $m$ from the neighborhood of the mass $m$ part of the relevant (product of) Lagrangian fields [12]. The asymptotic (in or out) fields for (possibly 
composite) particles are characterized by their rest energy $E$, mass $m$, and spin $J$. Suppressing the spin in what follows, we define the asymptotic fields associated with the interacting field $A(\mathbf{x}, t)$ as

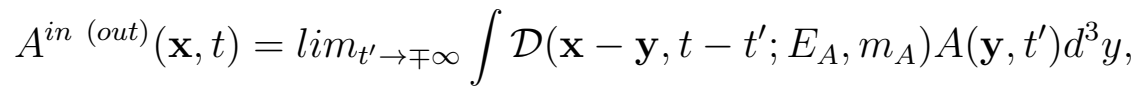

where the limit is the weak limit of the smeared operators, and $\mathcal{D}(\mathbf{x}, t)$ is defined in Eq.(9). The asymptotic fields for other interacting fields are defined in an analogous way. (We define the asymptotic fields for composite particles in Sec. 8.) The asymptotic limit in momentum space is often useful in calculations:

$$
\tilde{A}^{\text {in }{ }^{(o u t)}}(\mathbf{k}, E)=\lim _{t^{\prime} \rightarrow \mp \infty} \delta\left(E-\frac{\mathbf{k}^{2}}{2 m}\right) \int d E^{\prime} e^{i\left(E-E^{\prime}\right) t^{\prime}} \tilde{A}\left(\mathbf{k}, E^{\prime}\right)
$$

Either form of the definition of the asymptotic limits makes clear that asymptotic fields obey the free equations of motion:

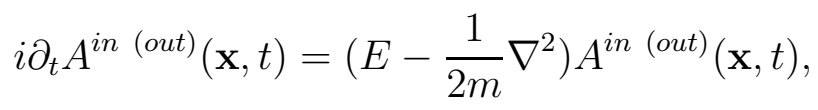

as well as the free field anticommutation or commutation relations:

$$
\left[A^{\text {in }(\text { out })}(\mathbf{x}, t), A^{\text {in }(\text { out })}\left(\mathbf{y}, t^{\prime}\right)\right]_{ \pm}=\mathcal{D}\left(\mathbf{x}-\mathbf{y}, t-t^{\prime}\right)
$$

Note that:

$$
\mathcal{D}(\mathbf{x}, 0)=\delta(\mathbf{x})
$$

for all $E, m$.

\section{Construction of asymptotic fields for bound states}

In this section, we show how to construct the asymptotic fields for the bound states of the hydrogen atom from a convolution of the wave function with products of electron and proton fields at separated points. Since the hydrogen atom is made of an electron and a proton and these fields anticommute, as an intuitive guess we start with one-half the commutator of the electron and proton fields,

$$
\frac{1}{2}[p(\mathbf{y}, t), e(\mathbf{x}, t)]_{-}=
$$




$$
\begin{aligned}
& \frac{1}{2}\left[p^{i n}(\mathbf{y}, t)+\sum_{n l m} \int \psi_{n l m}^{p}\left(\mathbf{y}-\mathbf{x}^{\prime}\right), e^{i n \dagger}\left(\mathbf{x}^{\prime}, t\right) h_{n l m}^{i n}\left(\frac{m \mathbf{x}^{\prime}+M \mathbf{y}}{m+M}, t\right) d^{3} x^{\prime}\right. \\
& \left.e^{i n}(\mathbf{x}, 0)+\sum_{n^{\prime} l^{\prime} m^{\prime}} \int \psi_{n l m}^{e}\left(\mathbf{x}-\mathbf{y}^{\prime}\right) p^{i n \dagger}\left(\mathbf{y}^{\prime}, t\right) h_{n^{\prime} l^{\prime} m^{\prime}}^{i n}\left(\frac{m \mathbf{x}+M \mathbf{y}^{\prime}}{m+M}\right) d^{3} y^{\prime}\right]_{-} .
\end{aligned}
$$

After normal-ordering, the terms linear in $h_{n^{\prime} l^{\prime} m^{\prime}}^{i n}$ are:

$$
\begin{gathered}
\sum_{n^{\prime} l^{\prime} m^{\prime}} \frac{1}{2}\left[\psi_{n^{\prime} l^{\prime} m^{\prime}}^{e}(\mathbf{x}-\mathbf{y})-\psi_{n^{\prime} l^{\prime} m^{\prime}}^{p}(\mathbf{y}-\mathbf{x})\right] h_{n^{\prime} l^{\prime} m^{\prime}}^{i n}\left(\frac{m \mathbf{x}+M \mathbf{y}}{m+M}\right)= \\
\sum_{n^{\prime} l^{\prime} m^{\prime}} \psi_{n^{\prime} l^{\prime} m^{\prime}}(\mathbf{x}-\mathbf{y}) h_{n^{\prime} l^{\prime} m^{\prime}}^{i n}\left(\frac{m \mathbf{x}+M \mathbf{y}}{m+M}\right),
\end{gathered}
$$

where we used Eq.(28). We can use the orthonormality of the hydrogen wave functions to eliminate the wave functions and isolate the in field for the states of the hydrogen atom: The term linear in the hydrogen in field becomes:

$$
\int \psi_{n l m}^{*}(\mathbf{x}-\mathbf{y}) \psi_{n^{\prime} l^{\prime} m^{\prime}}(\mathbf{x}-\mathbf{y}) h_{n^{\prime} l^{\prime} m^{\prime}}^{i n}\left(\frac{m \mathbf{x}+M \mathbf{y}}{m+M}\right) d^{3}(\mathbf{x}-\mathbf{y})=h_{n l m}^{i n}\left(\frac{m \mathbf{x}+M \mathbf{y}}{m+M}\right)
$$

Now we define an interpolating field for the hydrogen states,

$$
h_{n l m}(\mathbf{R}, t)=\frac{1}{2} \int d^{3} r \psi_{n l m}^{*}(\mathbf{r})\left[p\left(\mathbf{R}-\frac{m}{m+M} \mathbf{r}, t\right), e\left(\mathbf{R}+\frac{M}{m+M} \mathbf{r}, t\right)\right]_{-} .
$$

From these calculations, we see that the only term in this interpolating field that is linear in $h_{n l m}^{i n}$ is just $h_{n l m}^{i n}$, and further this is the only term with a singularity at the energy and mass of the bound state. Therefore this term can be isolated by taking the weak limit:

$$
h_{n l m}^{\text {in }(\text { out })}(\mathbf{R}, t)=\lim _{\tau \rightarrow \mp \infty} \int_{t^{\prime}=\tau} d^{3} R \mathcal{D}\left(\mathbf{R}-\mathbf{R}^{\prime}, t-t^{\prime} ; E_{n l m}, m+M\right) h_{n l m}\left(\mathbf{R}^{\prime}, t\right) .
$$

This discussion of bound states, which involves the bound-state amplitude, is an alternative to the constructions of bound-state operators from products constituent fields at a point that were formulated by Nishijima [8] and by Zimmermann [9].

\section{Summary}

We derived the Schrödinger equation for bound states of the hydrogen atom using the operator equation of motion for the electron (or proton) field together with the 
Haag expansion of the fields in terms of normal-ordered products of in (or out) fields. This was done without any reference to the Bethe-Salpeter equation. The usual normalization conditions and probability interpretation of bound state amplitudes are valid both in the present nonrelativistic example as well as for relativistic theories.

The $\mathrm{N}$ quantum approach has been applied to many other problems, and a survey of results appears in Ref.( [15]).

\section{Appendix: the asymptotic limits}

Here we discuss the asymptotic limits of the N-quantum approach, and indicate how the weak limits eliminate terms that do not have delta-function or principal-value singularities on the energy shell of the asymptotic field. Considering an operator $\mathcal{O}(\mathbf{x}, t)$, we define the asymptotic limits $\mathcal{O}^{\text {in }(\text { out })}(\mathrm{x}, t)$ by

$$
\mathcal{O}^{\text {in(out })}(\mathbf{x}, t)=\lim _{t^{\prime} \rightarrow \mp \infty} \int d^{3} x^{\prime} \mathcal{D}\left(\mathbf{x}-\mathbf{x}^{\prime}, t-t^{\prime} ; E, m\right) \mathcal{O}\left(\mathbf{x}^{\prime}, t^{\prime}\right),
$$

Using a four-dimensional Fourier transform,

$$
\mathcal{O}(\mathbf{x}, t)=\frac{1}{(2 \pi)^{4}} \int d \omega d^{3} k \tilde{\mathcal{O}}(\omega, \mathbf{k}) e^{-i \omega t+i \mathbf{k} \cdot \mathbf{x}},
$$

we find the momentum-space form of the weak asymptotic limit:

$$
\tilde{\mathcal{O}}^{i n(o u t)}(\omega, \mathbf{k})=\lim _{t^{\prime} \rightarrow \mp \infty} \delta\left(\omega-E-\frac{\mathbf{k}^{2}}{2 m}\right) \int d \omega^{\prime} e^{i\left(\omega-\omega^{\prime}\right) t^{\prime}} \tilde{\mathcal{O}}\left(\omega^{\prime}, \mathbf{k}\right)
$$

As a matrix element between two states, this gives a relation between distributions. If the matrix element of $\tilde{\mathcal{O}}\left(\omega^{\prime}, \mathbf{k}\right)$ on the right hand side is in $L^{1}$, the RiemannLebesgue lemma states that the term will vanish in this limit except when there is a singularity in the matrix element of $\tilde{\mathcal{O}}\left(\omega^{\prime}, \mathbf{k}\right)$ on the energy shell $\omega^{\prime}=E+\frac{\mathbf{k}^{2}}{2 m}$. The limit is finite if the singularity is either a delta function in $\omega^{\prime}-E-\frac{\mathbf{k}^{2}}{2 m}$ or has a principal value $1 /\left(\omega^{\prime}-E-\frac{\mathbf{k}^{2}}{2 m}\right)$. The limit diverges for stronger singularities on the energy shell. Therefore the requirement that the weak asymptotic limit exist

eliminates terms that do not have delta-function or principal-value singularities on the energy shell. Ref.([12]) gives further discussion of weak asymptotic limits. 


\section{Acknowledgements}

It is a pleasure to thank Dan-Olof Riska for his hospitality at the Helsinki Institute for Physics as well as for informative and stimulating discussions. I thank Steven Cowen, Paul Hoyer and Claus Montonen for their interest and for their helpful

comments. I give special thanks to Tom Ferbel for many excellent suggestions to improve the readability of this paper.

\section{References}

[1] R. Haag, "On quantum field theories," K. Dan Vidensk. Selsk. Mat-Fys. Medd. 29 (12) 1-32 (1955).

[2] C.N. Yang and D. Feldman, "The S-matrix in the Heisenberg Representation," Phys. Rev. 79, 972-978 (1950).

[3] A. Raychaudhuri, "On the Application of the N-Quantum Approximation Method to Bound State Problems," University of Maryland PhD Thesis (1977), pp 1-101. For the appliocation to an all-scalar model, Phys. Rev. D 18, 46584672 (1978).

[4] O.W. Greenberg and R. Genolio, "Introduction to the $N$-quantum approximation for bound states: the deuteron in pseudoscalar meson theory," Phys. Rev. 150, 1070-1076 (1966).

[5] F. Gross, "Three-dimensional covariant integral equations for low-energy systems," Phys.Rev. 186, 1448-1462 (1969).

[6] K. Gottfried and T.M. Yan, Quantum Mechanics: Fundamentals, Second Edition, (Springer, New York, 2004), pp 368-376.

[7] P.A.M. Dirac, "Gauge-Invariant Formulation of Quantum Electrodynamics," Can. J. Phys. 33, 650-660 (1955); M. Creutz, "Quantum Electrodynamics In The Temporal Gauge," Ann. Physics, 117, 471-483 (1979); A. Ilderton, M. Lavelle and D. McMullan, "Physical charges in QED and QCD," PoS QCDTNT09:019, 1-8, (2009) and related papers. 
[8] K. Nishijima, "Formulation of field theories of composite particles," Phys. Rev. 111, 995-1011 (1958).

[9] W. Zimmermann, "On the bound state problem in quantum field theory," Nuovo Cimento 10, 597-613 (1958).

[10] G. Källén, Quantum Electrodynamics, (Springer-Verlag, New York, 1972).

[11] H. Lehmann, K. Symanzik and W. Zimmermann, "On the formulation of quantized field theories" (in German) Nuovo Cimento 1, 205-225 (1955).

[12] O.W. Greenberg, "The asymptotic condition in quantum field theory," Princeton University PhD Thesis (1956), pp 1-220.

[13] S.R. Corley and O.W. Greenberg, "Bound states in Galilean-invariant field theory," J. Math. Phys. 38, 571-581 (1997).

[14] V. Bargmann, "On Unitary Ray Representations of Continous Groups," Annals of Mathematics 59, 1-46 (1997).

[15] O.W. Greenberg, "Virtues of the Haag expansion in quantum field theory," in Proc. of the First Arctic Workshop on Future Physics and Accelerators, eds. M. Chaichian, K. Huitu and R. Orava, (World Scientific, Singapore, 1995), pp 498-520. 